\title{
Outage and Bit Error Probability Analysis in Energy Harvesting Wireless Cooperative Networks
}

\author{
Ly Tran Thai Hoc ${ }^{1,2}$, Hoang-Sy Nguyen ${ }^{1,3}$, Quoc-Phu Ma ${ }^{1}$, Van Van Huynh ${ }^{4, *}$, \\ Thanh-Long Nguyen ${ }^{1,5}$, Huynh Tan Phuoc ${ }^{1}$, Miroslav Voznak ${ }^{1}$ \\ ${ }^{I}$ IT4Innovations, VSB - Technical University of Ostrava, \\ 17. listopadu 2172/15, Ostrava 708 00, Czech Republic \\ ${ }^{2}$ Nguyen Tat Thanh University, \\ Ho Chi Minh City, Vietnam \\ ${ }^{3}$ Faculty of Information Technology, Robotics, and Artificial Intelligence, Binh Duong University, \\ Thu Dau Mot City, Binh Duong Province, Vietnam \\ ${ }^{4}$ Modeling Evolutionary Algorithms Simulation and Artificial Intelligence, Faculty of Electrical \& \\ Electronics Engineering, Ton Duc Thang University, \\ Ho Chi Minh City, Vietnam \\ ${ }^{5}$ Center for Information Technology, Ho Chi Minh City University of Food Industry, \\ Ho Chi Minh City, Vietnam \\ huynhvanvan@tdtu.edu.vn
}

\begin{abstract}
This study focuses on a wireless powered cooperative communication network (WPCCN), which includes a hybrid access point (HAP), a source and a relay. The considered source and relay are installed without embedded energy supply (EES), thus are dependent on energy harvested from signals from the HAP to power their cooperative information transmission (IT). Taking inspiration from this, the author group investigates into a harvest-then-cooperate (HTC) protocol, whereas the source and the relay first harvest the energy from the AP in a downlink (DL) and then collaboratively work in uplink (UL) for IT of the source. For careful evaluation of the system performance, derivations of the approximate closed-form expression of the outage probability (OP) and an average bit error probability (ABER) for the HTC protocol over Rayleigh fading channels are done. Lastly, the author group performs Monte-Carlo simulations to reassure the numerical results they obtained.
\end{abstract}

Index Terms-Energy harvesting; Harvest-then-cooperate; Outage probability; Bit error probability.

\section{INTRODUCTION}

Recently, a wireless powered cooperative communication network (WPCCN) has been able to self-sustain their operation thanks to the new emerging approach, energy harvesting $(\mathrm{EH})$ [1]. In EH-based systems, wireless devices are used to harvest energy from their surroundings, for instance, wind, vibration, solar, and geothermal sources [2].

Manuscript received 14 February, 2019; accepted 25 July, 2019.

This work was supported by The Ministry of Education, Youth and Sports from the Large Infrastructures for Research, Experimental Development and Innovations project "IT4Innovations National Supercomputing Center" (No. LM2015070) and partially received a financial support from grant (No. SGS SP2019/41) conducted at VSB Technical University of Ostrava, Czech Republic.
Among others, radio frequency (RF) stands out to be a favourable energy source owing to its ease to control, availability and ability to carry energy and information simultaneously. To utilize those characteristics of RF, the author group in [3]-[5] invents namely simultaneous wireless information and power transfer (SWIPT) systems for the receiver architectures. SWIPT systems perform satisfactorily transmission in short range, but not in a long range. Thus, as a solution for this, the author group in [6] presents a novel wireless-powered system architecture, whereas wireless devices are powered by power-beacons (PBs). Followingly, a number of PB-assisted EH systems are proposed as in [7], [8].

In-depth studies about EH-based point-to-point systems have established a foundation for researchers to investigate further into more sophisticated system architectures, for instance, cognitive radios, cognitive relaying networks, and relay/cooperative systems. As for systems with relays, power splitting-based relaying (PSR) and time switching-based relaying (TSR) are the two most frequently used protocols for signal processing and $\mathrm{EH}$ at a specific relay node [9]. Moreover, a special TS-PS protocol, namely hybrid TS-PS relaying (HTPS) protocol, is studied in [10].

Additionally, throughput analysis results of decode-andforward (DF) and amplify-and-forward (AF) relaying systems are presented in [4], [9], and [11]. Furthermore, a number of different aspects related to EH relay systems are examined. For instance, EH-based relaying networks with interference [12], EH-based cooperative systems operating with spatially random relays [13], selection of relay for compromising the average energy and the information quality being transferred [14], and, last but not least, 
selection scheme of relay for hybrid full/half-duplex EHbased systems [15].

A harvest-then-transmit protocol has been developed recently. In this protocol, the energy of signals sent by an antenna of the HAP in the DL is collected. This energy is, then, used to send the independent information to the HAP in the UL via time-division-multiple-access (TDMA). A similar setup making use of a multi-antenna of the HAP is studied in [16], in which users concurrently are able to send information to the HAP in the UL utilizing space-divisionmultiple-access technique with the harvested energy in the DL previously. The users' minimum throughput is maximized with joint optimization of time allocation, UL transmit power allocation, DL energy beams, and the received beam-forming vectors. Latterly, for improving the performance of the multi-user wireless-powered communication network (WPCN), the full-duplex technique is implemented as mentioned in [17], [18]. This technique utilizes two antennas for two purposes: sending DL users' energy via wireless connection and, simultaneously, receiving UL users' information by means of TDMA.

Different from the works mentioned above, the authors of this paper consider distributed PBs in WPCCN, which assists the HTC protocol, in this study. Particularly, the used time-switching protocol consisting of UL wireless information transmission (WIT) and DL wireless energy transmission (WET) are deployed. One sustained power supply is in connection with the HAP. The source and the relay are not installed with any EES, but rechargeable batteries. Thus, the RF energy transmitted from the HAP can be harvested and stored. The primary contribution of this study can be summarized as follows:

- Derivation of analytical expressions for OP implementing the AF relaying protocol and selecting techniques, respectively, on the relay and the HAP;

- The approximately derived closed-form expression of the ABER of the proposed HTC protocol in the Rayleigh fading channels.

The rest of the paper is organized as follows. In Section II, we give descriptions of the system model and the HTC protocol. In Section III, analysis of approximate closed-form expression for the OP and ABER is provided. In Section IV, simulation results and discussions are presented. Finally, conclusions are given in Section V.

\section{SySTEM MOdEL}

Figure 1 illustrates the network under consideration. In the figure, there are a HAP (B), a(n) (information) source (A), and a relay $(\mathrm{R})$. The relay receives no traffic and assists the source in IT process. Each node is assumed to have one antenna and operates in a half-duplex mode via an AF relay. Besides, the relay and the source are installed without EES. Hence, firstly, the signal energy sent from the HAP to the DL is harvested. Then, it is stored in rechargeable batteries and is ready to be used for the IT operation to the HAP in the UL.

It is denoted that $h_{X Y}$ is the channel coefficients of $X \rightarrow Y$ links knowing that $X, Y \in\{A, R, B\}$. Additionally, the channel power gain $\left|h_{X Y}\right|^{2}$ is the random variables (RVs), which is distributed exponentially with mean value of $\Omega_{X Y}=\left(d_{X Y}\right)^{-\zeta}$, whereas $d_{X Y}$ is the distance of $X \rightarrow Y$ links. The path-loss exponent is denoted as $\varsigma$. Last but not least, channels in DL and UL are assumed to undergo independently slow and flat fading frequency. The channel gains for each transmission block denoted as $T$ is constant, but varies independently on different blocks.

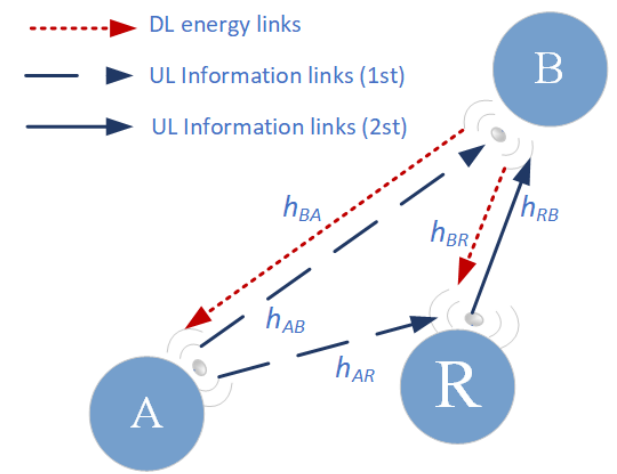

Fig. 1. System model.

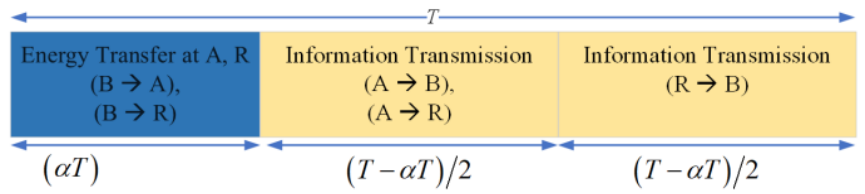

Fig. 2. The HTC protocol.

Figure 2 illustrates the HTC protocol of the considered network. The transmission duration $T$ is divided orderly into three blocks. The first block, $\alpha T$, is the DL energy transferred from the HAP to the source and the relay with time switching (TS) ratio of $\alpha \in(0,1)$. The remaining time is equally divided into two blocks being $(1-\alpha) T / 2$, which are allocated to the cooperative operation of IT in the UL. In the first block for the UL, data is transmitted to the HAP by the source utilizing the stored harvested energy. It is noted that the relay can overhear this data owing to the wireless communication broadcasting feature. In the second block for the UL, the relay implementing the AF relaying protocol, which appears to be less complex [9], uses the stored harvested energy during the DL to assist the transmitting information from the source.

It is noted that for the information processing operation in the HAP, the selection combining (SC) technique in [19] is chosen. Utilizing the SC technique also helps to reveal the system closed form analysis, which is tractable. Operation wise, the HAP information receiver first compares the signals sent by the source and the relay, after that, picks the one with larger signal-to-noise ratio (SNR). Then, it is used to decode the information of the source by the HAP in the end of each time block.

The harvested energy amount from the source and the relay are calculated as in [9], [15]:

$$
E_{A}=\eta \alpha P_{B}\left|h_{B A}\right|^{2} T
$$




$$
E_{R}=\eta \alpha P_{B}\left|h_{B R}\right|^{2} T
$$

where $P_{B}$ is the HAP transmission power during the DL, which is assumed to be adequately large, so that the harvested energy from the noise can be neglected. The RFto-DC energy conversion efficiency is $\eta \in(0,1)$. Utilizing the rectenna technology as in [20], fairly high efficiency (e.g., $80 \%$ ) can be achieved.

Thus, the transmitted power of the source and the relay during the UL are calculated as follows:

$$
\begin{gathered}
P_{A}=2 E_{A} /(1-\alpha) T=2 \eta \alpha P_{B}\left|h_{B A}\right|^{2} /(1-\alpha), \text { and } \\
P_{R}=2 E_{R} /(1-\alpha) T=2 \eta \alpha P_{B}\left|h_{B R}\right|^{2} /(1-\alpha) .
\end{gathered}
$$

The received SNR at the HAP after the transmission of the sources, thus, can be expressed as

$$
\gamma_{A}=P_{B}\left|h_{B A}\right|^{2} / N_{0}=\chi \delta\left|h_{B A}\right|^{2}\left|h_{A B}\right|^{2},
$$

where $\chi=2 \eta \alpha /(1-\alpha), \delta=P_{B} / N_{0}$, and $N_{0}$ is the power of the noise subjected to all the receivers.

As mentioned earlier, the relay can overhear the signal sent from the source. Thus, in this second time block of the UL, the received signal is amplified and forwarded to the HAP utilizing the power $P_{R}$ as mentioned in (3) with the amplification factor of $\beta=\sqrt{1 /\left(\delta\left|h_{A R}\right|^{2}+1\right)}$ [4], [9]. After manipulating, the received SNR at the HAP from the link A$\mathrm{R}-\mathrm{B}$ can be expressed as follows

$$
\gamma_{A R B}=\frac{\chi \delta\left|h_{B A}\right|^{2}\left|h_{A R}\right|^{2} \chi \delta\left|h_{B R}\right|^{2}\left|h_{R B}\right|^{2}}{\chi \delta\left|h_{B A}\right|^{2}\left|h_{A R}\right|^{2}+\chi \delta\left|h_{B R}\right|^{2}\left|h_{R B}\right|^{2}+1} .
$$

Remark 1: As can be observed in (5) - the resulting SNR, the closed-form expression of the exact value of OP is difficult to calculate. Thanks to [21], the SNR in terms of $\gamma_{A R B}$ given in (6) can be approximated as

$$
\begin{aligned}
\gamma_{A R B} & \approx \frac{\chi \delta\left|h_{B A}\right|^{2}\left|h_{A R}\right|^{2} \chi \delta\left|h_{B R}\right|^{2}\left|h_{R B}\right|^{2}}{\chi \delta\left|h_{B A}\right|^{2}\left|h_{A R}\right|^{2}+\chi \delta\left|h_{B R}\right|^{2}\left|h_{R B}\right|^{2}} \leq \\
& \leq \chi \delta \min \left(\left|h_{B A}\right|^{2}\left|h_{A R}\right|^{2},\left|h_{B R}\right|^{2}\left|h_{R B}\right|^{2}\right) .
\end{aligned}
$$

As the SC technique is implemented on the HAP receiver, the received SNR at the HAP node for the HTC protocol is given by

$$
\gamma_{B}=\max \left(\gamma_{A B}, \gamma_{A R B}\right)
$$

The achievable rate of transmitted information between the HAP and the source via R is expressed as follows

$$
I_{B}=\frac{(1-\alpha)}{2} \log _{2}\left(1+\gamma_{B}\right) \text {. }
$$

\section{Outage Probability AND AVERAge Bit ERror PROBABILITY ANALYSIS}

This section shows the analytical formulation of the OP and ABER, respectively.

\section{A. The Outage Probability}

The OP stands for the instantaneous received SNR, $\gamma_{A}$ value under a threshold value of $\gamma_{0}=2^{2 R_{0}}-1$, noting that $R_{0}$ is the source (fixed) transmission rate. In addition, the Remark 1 leads to the Theorem 1 .

Theorem 1. The approximate expression of the OP of the HTC protocol can be given as follows

$$
O P \approx A_{1}-A_{2} \times\left(A_{3 a}-A_{3 b}\right),
$$

whereas:

$$
\begin{aligned}
A_{1}= & 1-\left(\sqrt{\frac{4 \gamma_{0}}{\chi \delta \Omega_{B A} \Omega_{A B}}}\right) K_{1}\left(\sqrt{\frac{4 \gamma_{0}}{\chi \delta \Omega_{B A} \Omega_{A B}}}\right), \\
A_{2} & =\left(\sqrt{\frac{4 \gamma_{0}}{\chi \delta \Omega_{B R} \Omega_{R B}}}\right) K_{1}\left(\sqrt{\frac{4 \gamma_{0}}{\chi \delta \Omega_{B R} \Omega_{R B}}}\right), \\
A_{3 a} & =\left(\sqrt{\frac{4 \gamma_{0}}{\chi \delta \Omega_{B A} \Omega_{A R}}}\right) K_{1}\left(\sqrt{\frac{4 \gamma_{0}}{\chi \delta \Omega_{B A} \Omega_{A R}}}\right), \\
A_{3 b} & =\left(\sqrt{\frac{4 \gamma_{0}}{\chi \delta \Omega_{B A} \Omega_{A R}}+\frac{4 \gamma_{0}}{\chi \delta \Omega_{B A} \Omega_{A B}}}\right) \times \\
& \times K_{1}\left(\sqrt{\frac{4 \gamma_{0}}{\chi \delta \Omega_{B A} \Omega_{A R}}+\frac{4 \gamma_{0}}{\chi \delta \Omega_{B A} \Omega_{A B}}}\right),
\end{aligned}
$$

and $K_{1}($.$) denoting the second kind of modified Bessel$ function with the first order [21].

Prooffor Theorem 1: Based on the definition of the OP

$$
O P=\operatorname{Pr}\left(\gamma_{A}<\gamma_{0}\right)=\operatorname{Pr}\left(\gamma_{A B}<\gamma_{0}, \gamma_{A R B}<\gamma_{0}\right) .
$$

It should be noted that there is a correlation between the SNRs $\gamma_{A B}$ and $\gamma_{A R B}$ as they both contain the random variable $h_{B A}$. Accordingly, the following expression can be obtained

$$
\operatorname{Pr}\left(\gamma_{A B}<\gamma_{0}, \gamma_{A R B}<\gamma_{0}\right) \neq \operatorname{Pr}\left(\gamma_{A B}<\gamma_{0}\right) \operatorname{Pr}\left(\gamma_{A R B}<\gamma_{0}\right) .
$$

This is on the contrary to the conventional constantpowered cooperative networks, in which different paths SNRs are usually independent. Because of the intricate $\gamma_{A R B}$ structure and the mentioned correlation, the closed-form expression of (5) is difficult to acquire. The approximate SNR value, according to the Remark 1 , is easier to tract than the exact value. Hence, it assists deriving the closed-form expression of the OP. Consequently, the OP (14) can be approximately expressed by

$$
\begin{gathered}
O P \approx \operatorname{Pr}\left(\begin{array}{l}
\left|h_{B A}\right|^{2}\left|h_{A B}\right|^{2}<\gamma_{0} / \chi \delta, \\
\min \left(\left|h_{B A}\right|^{2}\left|h_{A R}\right|^{2},\left|h_{B R}\right|^{2}\left|h_{R B}\right|^{2}\right)<\gamma_{0} / \chi \delta
\end{array}\right)= \\
=\operatorname{Pr}\left(\left|h_{B A}\right|^{2}\left|h_{A B}\right|^{2}<\gamma_{0} / \chi \delta\right)- \\
-\operatorname{Pr}\left(\begin{array}{l}
\left|h_{B A}\right|^{2}\left|h_{A B}\right|^{2}<\gamma_{0} / \chi \delta, \\
\min \left(\left|h_{B A}\right|^{2}\left|h_{A R}\right|^{2},\left|h_{B R}\right|^{2}\left|h_{R B}\right|^{2}\right)>\gamma_{0} / \chi \delta
\end{array}\right) .
\end{gathered}
$$

Taking into account that $h_{B R}$ and $h_{R B}$ are independent of 
$h_{B A} \quad$ as in $[22, \quad$ p. 21]; the fact that $\operatorname{Pr}(A, B)=\operatorname{Pr}(A)-\operatorname{Pr}(A, \bar{B})$, whereas $\operatorname{Pr}(A)$, denotes the probability of event $A$, and $\operatorname{Pr}(A, \bar{B})$ denotes the probability of the event $A$ and $B$. The following expression can be obtained as

$$
\begin{aligned}
O P & \approx \underbrace{\operatorname{Pr}\left(\left|h_{B A}\right|^{2}\left|h_{A B}\right|^{2}<\gamma_{0} / \chi \delta\right)}_{I}-\underbrace{\operatorname{Pr}\left(\left|h_{B R}\right|^{2}\left|h_{R B}\right|^{2}>\gamma_{0} / \chi \delta\right)}_{I_{2}} \times \\
& \times \underbrace{\operatorname{Pr}\left(\left|h_{B A}\right|^{2}\left|h_{A B}\right|^{2}<\gamma_{0} / \chi \delta,\left|h_{B A}\right|^{2}\left|h_{A R}\right|^{2}>\gamma_{0} / \chi \delta\right)}_{I_{3}} .
\end{aligned}
$$

Firstly, the $I_{1}$ can be written as

$$
I_{1}=1-\frac{1}{\Omega_{A B}} \int_{x=0}^{\infty} e^{-\frac{\gamma_{0}}{\chi \delta \Omega_{B A} x}-\frac{x}{\Omega_{A B}}} d x=A_{1},
$$

whereas the integral identity is solved with [21, Equation (3.324.1)].

Likewise, it can be noted from $I_{2}$ that

$$
I_{2}=1-\frac{1}{\Omega_{R B}} \int_{x=0}^{\infty} e^{-\frac{\gamma_{0}}{\chi \delta \Omega_{B R} x}-\frac{x}{\Omega_{R B}}} d x=A_{2} .
$$

Eventually, the $I_{3}$ can be written as

$$
\begin{aligned}
& I_{3}=\int_{x=0}^{\infty} \operatorname{Pr}\left(\left|h_{A B}\right|^{2}<\frac{\gamma_{0}}{\chi \delta x},\left|h_{A R}\right|^{2}>\frac{\gamma_{0}}{\chi \delta x}\right) f_{\left|h_{B A}\right|^{2}}(x) d x= \\
& =\int_{x=0}^{\infty} \operatorname{Pr}\left(\left|h_{A R}\right|^{2}>\frac{\gamma_{0}}{\chi \delta x}\right) \operatorname{Pr}\left(\left|h_{A B}\right|^{2}<\frac{\gamma_{0}}{\chi \delta x}\right) f_{\left|h_{B A}\right|^{2}}(x) d x .
\end{aligned}
$$

The simplification of the equation (16) can be expressed as

$$
\begin{gathered}
I_{3}=\frac{1}{\Omega_{B A}} \int_{x=0}^{\infty}\left(e^{-\frac{\gamma_{0}}{\chi \delta \Omega_{A R} x}-\frac{x}{\Omega_{B A}}}\right) d x- \\
-\frac{1}{\Omega_{B A}} \int_{x=0}^{\infty} e^{-\frac{1}{x}\left(\frac{\gamma_{0}}{\chi \delta \Omega_{A B}}+\frac{\gamma_{0}}{\chi \delta \Omega_{A R}}\right)-\frac{x}{\Omega_{B A}}} d x= \\
=A_{3 a}-A_{3 b} .
\end{gathered}
$$

As (18), (19), and (21) are substituted into (17), the desired result as in (9) can be obtained. The proof is subsequently completed.

Remark 2: The relay performs two tasks: source signal energy harvesting and forwarding that signal to a destination. From Theorem 1, the destination OP is derived. In (9), OP function is dependent on the $\mathrm{EH}$ time, $\alpha$. As $\alpha$ value increases from 0 to 1 , OP value decreases. Since $\alpha$ becomes larger, the relay gains more transmission power. Thus, the outage chance decreases. Further discussions can be found in the numerical results session below.

\section{B. Average Bit Error Probability}

The ABER used for evaluating the wireless communication performance is studied in this subsection. It is noted that the evaluation is done with different, widely used modulations. In particular, there are BFSK, BPSK transmitting orthogonal signal, and $M$-ary square QAM alphabet transmitting data by changing the two carriers' signal amplitudes.

So, as to obtain the ABER as mentioned in [23], the below expression is considered

$$
B=\mathbb{E}|a Q(\sqrt{2 b \gamma})|,
$$

whereas $\mathbb{E}\{|\cdot|\}$ is the expectation operator, $Q(x)$ is the Gaussian Q-function defined as $Q(x)=\frac{1}{\sqrt{2 \pi}} \int_{x}^{\infty} e^{-t^{2} / 2} d t$, $(a, b)=(1,2)$ for BPSK, and $(a, b)=(1,1)$ for QPSK.

Founded on the OP approximated expression mentioned in (9) and the ABER expression in (22), a corollary of the HTC protocol ABER can be obtained.

Corollary 1. Expression of approximate closed-form of the HTC protocol ABER value can be written as follows

$$
B=\frac{a \sqrt{b}}{2 \sqrt{\pi}} \int_{\gamma=0}^{\infty} \frac{e^{-b \gamma}}{\sqrt{\gamma}} O P(\gamma) d \gamma .
$$

\section{NUMERICAL RESULTS}

In this section, numerical results for validation of the OP and ABER calculations acquired in Section III are provided and some system performance insights are given. Moreover, the comparison between the aforementioned expressions and the complementary Monte Carlo-simulated performance results for the accuracy evaluation is done. Specifically, there are $10^{6}$ Rayleigh distribution random variables generated. The average power represents the distances between all links. For the ease of the calculation, a linear topology, whereas the relay, the source, and the HAP are assumed to be located on a straight line, is considered, i.e., $d_{R B}=d_{A B}-d_{A R}$. In simulations done hereafter, the following parameters $d_{A B}=1, d_{A R}=0.3, \zeta=3, \eta=50 \%$, $\alpha=1 / 4$, and $R_{0}=1(\mathrm{~dB})$ are set.

Figure 3 depicts the OP performance against the transmit SNR $\delta(\mathrm{dB})$ considering different distance values between $\mathrm{R}$ and $\mathrm{B}, d_{R B}=0.3, d_{R B}=0.5$, and $d_{R B}=0.7$. The simulation results, as can be observed, match perfectly to the analytical ones acquired in Section III. Additionally, as the R-B link quality decreases, the OP for IT in (14) increases. Nevertheless, the gap between the corresponding curves increases as $d_{R B}$ value decreases from 0.7 to 0.5 and, then, to 0.3 . This indicates that for a shorter distance of the S-R link, there is a higher amount of energy harvested by the relay assisting the HTC protocol.

Figure 4 represents the transmission rate versus the TS ratio, $\alpha$ with different transmit SNR values, $\delta(\mathrm{dB})$. It can be observed that as $\alpha$ increases from 0 to an optimal value (approximately 0.3), so does the transmission rate. However, the curve starts decreasing as $\alpha$ continues increasing to its optimal. This can be explained as when $\alpha$ is less than its optimal value, there is less time for the system to harvest the energy. Additionally, the SNR increases in conjunction with the transmission rate. As can apparently be observed, in case 
of $\delta=10(\mathrm{~dB})$, the system has a better transmission rate comparing to $\delta=5(\mathrm{~dB})$.

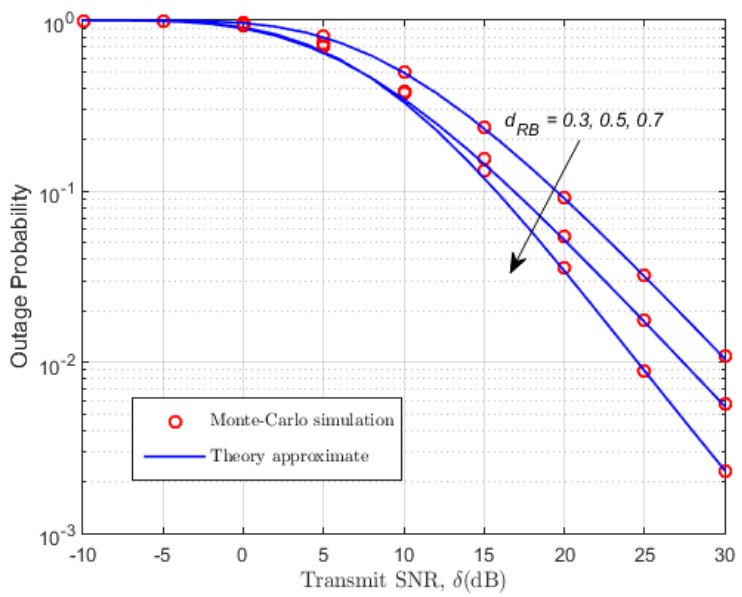

Fig. 3. The OP versus transmit SNR, $\delta(\mathrm{dB})$.

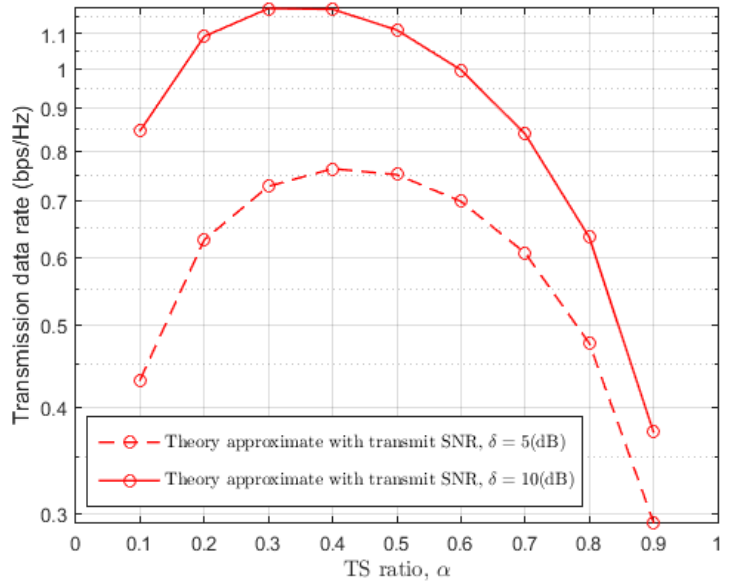

Fig. 4. The transmission rate versus TS ratio, $\alpha$.

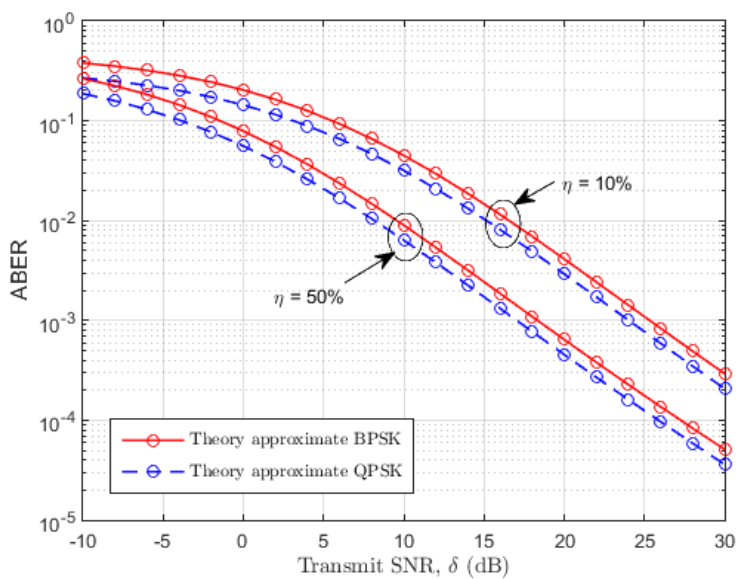

Fig. 5. The ABER rate versus transmit SNR, $\delta(\mathrm{dB})$ with different $\mathrm{EH}$ efficiency values $\eta=10 \%$ and $\eta=50 \%$.

Figure 5 illustrates the ABER function in relation with the transmit SNR in two modulations: BPSK and QPSK, i.e., $(a=b=1)$ and $(a=1, b=2)$, respectively. This functions with different values of the EH efficiency; $\eta=10 \%$ and $\eta=50 \%$ are plotted for the comparison. The ABER is dependent on the transmit power of the source and the relay. It should be noted that the ABER in BPSK scheme is always better than that of the QPSK. The ABER of the systems increases linearly as the conversion efficiency $\eta$ increases from $10 \%$ to $50 \%$.

\section{CONCLUSIONS}

In this study, the author group focuses on a HTC protocol being the WPCCN. In this network, there are two pieces of energy-constrained equipment: a source and a relay. They operate on the energy harvested from the received RF signal and utilize that to assist forwarding the source signal to the HAP. By utilizing this technology, a relatively high value of efficiency, $80 \%$, can be achieved. The TS architecture is adopted to build the above network. Moreover, the closedform expression of the approximated OP and the ABER in Rayleigh-fading channel for the mentioned protocol are derived. Nevertheless, the effect of the varied system parameters on the HTC protocol performance utilizing AF relay is shown with numerical analyses throughout the paper, whereas the optimal value for TS ratio is approximately 0.3 .

\section{REFERENCES}

[1] X. Lu, P. Wang, D. Niyato, D. I. Kim, and Z. Han, "Wireless networks with RF energy harvesting: a contemporary survey", IEEE Communications Surveys and Tutorials, vol. 17, no. 2, pp. 757-789, 2015. DOI: 10.1109/COMST.2014.2368999.

[2] S. Sudevalayam and P. Kulkarni, "Energy harvesting sensor nodes: Survey and implications", IEEE Communications Surveys and Tutorials, vol. 13, no. 3, pp. 443-461, 2011. DOI: 10.1109/SURV.2011.060710.00094.

[3] R. Zhang and C. K. Ho, "MIMO broadcasting for simultaneous wireless information and power transfer", IEEE Trans. Wirel. Commun., vol. 12, no. 5, pp. 1989-2001, 2013. DOI: 10.1109/TWC.2013.031813.120224.

[4] H. -S. Nguyen, M. Voznak, M. -T. Nguyen, and L. Sevcik, "Performance analysis with wireless power transfer constraint policies in full-duplex relaying networks", Elektronika ir Elektrotechnika vol. 23, no 4, pp. 70-76, 2017. DOI: 10.5755/j01.eie.23.4.18725.

[5] T. X. Doan, T. M. Hoang, T. Q. Duong, and H. Q. Ngo, "Energy harvesting-based D2D communications in the presence of interference and ambient RF sources", IEEE Access, vol. 5, pp. 5224-5234, 2017. DOI: 10.1109/ACCESS.2017.2681696.

[6] K. Huang and V. K. N. Lau, "Enabling wireless power transfer in cellular networks: Architecture, modeling and deployment", IEEE Trans. Wirel. Commun., vol. 13, no. 2, pp. 902-912, 2014. DOI: 10.1109/TWC.2013.122313.130727.

[7] C. Zhong, X. Chen, Z. Zhang, and G. K. Karagiannidis, "Wirelesspowered communications: Performance analysis and optimization", IEEE Trans. Commun., vol. 63, no. 12, pp. 5178-5190, 2015. DOI: 10.1109/TCOMM.2015.2488640.

[8] Y. Ma, H. Chen, Z. Lin, Y. Li, and B. Vucetic, "Distributed and optimal resource allocation for power beacon-assisted wirelesspowered communications", IEEE Trans. Commun., vol. 63, no. 10 pp. 3569-3583, 2015. DOI: 10.1109/TCOMM.2015.2468215.

[9] A. A. Nasir, X. Zhou, S. Durrani, and R. A. Kennedy, "Relaying protocols for wireless energy harvesting and information processing", IEEE Trans. Wirel. Commun., vol. 12, no. 7, pp. 3622-3636, 2013 DOI: 10.1109/TWC.2013.062413.122042.

[10] H. S. Nguyen, T. S. Nguyen, and M. Voznak, "Successful transmission probability of cognitive device-to-device communications underlaying cellular networks in the presence of hardware impairments", EURASIP Journal on Wireless Communications and Networking, art. no. 208, 2017. DOI: 0.1186/s13638-017-0994-0.

[11] Z. Hadzi-Velkov, N. Zlatanov, T. Q. Duong, and R. Schober, "Rate maximization of decode-and-forward relaying systems with RF energy harvesting", IEEE Communications Letters, vol. 19, no. 12, pp. 2290-2293, 2015. DOI: 10.1109/LCOMM.2015.2489213.

[12] G. Zhu, C. Zhong, H. A. Suraweera, G. K. Karagiannidis, Z. Zhang, and T. A. Tsiftsis, "Wireless information and power transfer in relay systems with multiple antennas and interference", IEEE Trans. Commun., vol. 63, no. 4, pp. 1400-1418, 2015. DOI: 
10.1109/TCOMM.2015.2398862.

[13] Z. Ding, I. Krikidis, B. Sharif, and H. V. Poor, "Wireless information and power transfer in cooperative networks with spatially random relays", IEEE Trans. Wirel. Commun., vol. 13, no. 8, pp. 4440-4453, 2014. DOI: 10.1109/TWC.2014.2314114.

[14] H. S. Nguyen, T. S. Nguyen, and M. Voznak, "Relay selection for SWIPT: Performance analysis of optimization problems and the trade-off be-tween ergodic capacity and energy harvesting", $A E U$ International Journal of Electronics and Communications, vol. 85 , pp. 59-67, 2018. DOI: 10.1016/j.aeue.2017.12.012.

[15] H. S. Nguyen, T. S. Nguyen, V. T. Vo, and M. Voznak, "Hybrid fullduplex/half-duplex relay selection scheme with optimal power under individual power constraints and energy harvesting", Computer Communications, vol. 124, pp. 31-44, 2018. DOI: 10.1016/j.comcom.2018.04.014

[16] L. Liu, R. Zhang, and K. -C. Chua, "Multi-antenna wireless powered communication with energy beamforming", IEEE Trans. Commun., vol. 62, no. 12, pp. 4349-4361, 2014. DOI: 10.1109/TCOMM. 2014.2370035

[17] H. Ju and R. Zhang, "Optimal resource allocation in full-duplex wireless powered communication network", IEEE Trans. Commun. vol. 62, no. 10, pp. 3528-3540, 2014. DOI: 10.1109/TCOMM.2014.2359878.

[18] X. Kang, C. K. Ho, and S. Sun, "Full-duplex wireless-powered communication network with energy causality", IEEE Trans. Wirel. Commun., vol. 14, no. 10, pp. 5539-5551, 2015. DOI: 10.1109/TWC.2015.2439673.

[19] M. K. Simon and M. -S. Alouini, Digital communication over fading channels. New York, John Wiley and Sons, 2000. DOI: $10.1002 / 0471200697$

[20] N. Shinohara, Wireless Power Transfer Via Radiowaves. John Wiley \& Sons, 2014. DOI: 10.1002/9781118863008.

[21] I. S. Gradshteyn and I. M. Ryzhik, Table of integrals, series, and products. Elsevier, 2007. DOI: 10.1016/C2010-0-64839-5.

[22] A. Papoulis and S. U. Pillai, Probability, random variables, and stochastic processes. New York, McGraw-Hill, 2002.

[23] K. Cho and D. Yoon, "On the general BER expression of one- and two-dimensional amplitude modulations", IEEE Trans. Commun. vol. 50, no. 7, pp. 1074-1080, 2002. DOI: 10.1109/TCOMM.2002.800818 